\title{
Épiscopat canadien et collégialité
}

\section{Nive Voisine}

Volume 50, numéro 1, 1983

Bilan de l'histoire religieuse au Canada

Canadian Catholic History: A survey

URI : https://id.erudit.org/iderudit/1007039ar

DOI : https://doi.org/10.7202/1007039ar

Aller au sommaire du numéro

Éditeur(s)

Les Éditions Historia Ecclesiæ Catholicæ Canadensis Inc.

ISSN

0318-6172 (imprimé)

1927-7067 (numérique)

Découvrir la revue

Citer cet article

Voisine, N. (1983). Épiscopat canadien et collégialité. Sessions d'étude - Société canadienne d'histoire de l'Église catholique, 50(1), 119-140.

https://doi.org/10.7202/1007039ar

Tous droits réservés @ Les Éditions Historia Ecclesiæ Catholicæ Canadensis Inc., 1983
Ce document est protégé par la loi sur le droit d'auteur. L'utilisation des services d'Érudit (y compris la reproduction) est assujettie à sa politique d'utilisation que vous pouvez consulter en ligne.

https://apropos.erudit.org/fr/usagers/politique-dutilisation/ 


\section{Épiscopat canadien et collégialité*}

C'est le concile de Vatican II qui a vraiment mis en lumière la collégialité épiscopale. En effet, la constitution apostolique Lumen Gentium a rappelé «le caractère et la nature collégiale de l'ordre épiscopal que, d'ailleurs, dit-il, les Conciles oecuméniques réunis au cours des siècles confirment jusqu'à l'évidence", mais il a précisé aussitôt que "ce même pouvoir collégial peut être exercé, en union avec le Pape, par les évêques répandus en tous les points du monde (Lumen Gentium, 22.6)». De façon plus concrète, le décret Christu Dominus a souligné que

dès les premiers siècles de l'Église, la communion de la charité fraternelle et le souci de la mission universelle confiée aux Apôtres ont poussé les Évêques, placés à la tête des Églises particulières, à associer leurs forces et leurs volontés en vue de promouvoir le bien commun de l'ensemble des évêques et de chacune d'elles (Christus Dominus, 36).

et que:

De notre temps surtout, il n'est pas rare que les Évêques ne puissent accomplir leur charge convenablement et avec fruit s'ils ne réalisent pas avec les autres Évêques une concorde chaque jour plus étroite et une action plus coordonnée (ibid., 37).

Enfin, le motu proprio Ecclesiae Sanctae du 6 août 1966 a fait connaître les normes d'application des documents précédents ${ }^{1}$.

* Ce texte a été fait conjointement avec Jean Hamelin. Je l'ai rédigé entièrement, mais, pour la partie concernant le $\mathrm{XX}^{\mathbf{e}}$ siècle, mon collègue a assuré toute la recherche. Il m'a également permis de puiser largement dans le manuscrit du volume III de l'Histoire du catholicisme québécois, qu'il a rédigé en collaboration avec Nicơle Gagnon (pour le premier tome) et qui doit paraître en mai 1984 chez Boréal Express. Je suis néanmoins responsable des interprétations et des erreurs. N.V.

${ }^{1}$ Pour construire l'Église nouvelle: normes d'applications du Concile Vatican II et documents complementaires, Montréal, Fides, 1969, pp. 305-39; je cite Lumen Gentium et Christus Dominus d'après Paul-Aimé Martin, dir., Vatican II: les seize documents conciliaires, texte intégral, 2e éd., Montréal, Fides, 1967. Voir aussi Bernard A. Prince, «Episcopal Conferences and Collegiality», Le séminaire, vol. 34, no 1 (fév. 1969), pp. 27-33. 
Les déclarations du concile comportent sans doute un aspect innovateur, particulièrement à propos de «l'exercice régional de la solidarité épiscopale" ${ }^{2}$, mais elles ont aussi rappelé l'ancienneté du pouvoir collégial et souligné l'importance, en ce sens, des synodes, des conciles provinciaux et des conciles pléniers qui apparaissent dès les premiers siècles de l'Église et, plus récemment, des conférences épiscopales qui, selon Lumen Gentium, "peuvent aujourd'hui contribuer de façon multiple et efficace à aiguiller le sentiment collégial vers des réalisations concrètes» (Lumen Gentium, 23). Cette collégialité a été vécue de façon différente - du moins en intensité - dans les Églises particulières; c'est pourquoi il peut être intéressant de revoir le passé de certaines d'entre elles dans cette perspective précise.

De ce point de vue, l'expérience canadienne m'apparaît passionnante à étudier. L'immensité du territoire et la dispersion de la population ont contribué à isoler les évêques les uns des autres et à rendre difficile le travail collégial; d'autre part, le «fait franco-anglais», que Paul E. Crunigan appelle «le fait vital dominant de l'histoire [religieuse] du Canada" ${ }^{3}$, a accentué davantage les problèmes d'unité et d'action commune. En conséquence, nous pouvons nous poser la question suivante: dans quelle mesure les évêques du Canada ont-ils été capables de surmonter ces obstacles géographiques, culturels, linguistiques et autres pour inventer des formules de travail collégial? À première vue - et c'est mon hypothèse de départ - il m'apparaît que les chefs religieux canadiens, comme les hommes politiques d'ailleurs, ont peu travaillé collégialement et n'ont pas vraiment réussi à briser la barrière des «deux solitudes".

Pour répondre à la question posée et infirmer ou confirmer ma première intuition, je vais étudier d'abord ce que j'appellerais volontiers le travail collégial à l'ancienne mode, c'est-à-dire essentiellement celui qu'on retrouve dans les conciles (provinciaux et pléniers) et dans les assemblées d'évêques; dans une seconde partie, $\mathrm{j}$ 'insisterai davantage sur une forme plus moderne de collégialité régionale, celle des conférences épiscopales.

\footnotetext{
${ }^{2}$ J. Hamer, "Les conférences épiscopales, exercice de la collégialité », Nouvelle revue théologique, t. 85, no 9 (nov. 1963), p. 967.

${ }^{3}$ Paul E. Crunigan, «L'Église au Canada de 1850 à nos jours", Roger Aubert et al., L'Église dans le monde moderne, Paris, Le Seuil, 1975, p. 256.
} 


\section{LE TRAVAIL COLLÉGIAL DES ÉVÊQUES CANADIENS AVANT LA CONFÉRENCE CATHOLIQUE CANADIENNE}

Dès sa fondation, l'Église catholique canadienne couvre tout le territoire de l'Amérique du Nord - en principe, de l'Arctique à la Louisiane et de l'Atlantique au Pacifique - et un seul évêque est chargé de cet immense diocèse. La seule collégialité possible est celle qui unit le prélat à son clergé et elle se traduit par la tenue de synodes en 1690 , 1694,1698 et 1700 , pendant l'administration de $\mathrm{M}^{\mathrm{gr}}$ de Saint-Vallier.

Après la conquête, la juridiction de l'évêque de Québec se rétrécit pour se confondre presque avec les limites du Canada actuel: par exemple, le premier diocèse américain, Baltimore, est érigé en 1789 et il s'occupe de l'Illinois; la Louisiane est cédée aux Espagnols, les îles Saint-Pierre et Miquelon à la France; Terre-Neuve devient préfecture apostolique à partir de 1784 ; la Nouvelle-Écosse échappe au contrôle de Québec en 1816; quant à la côte du Pacifique, les incertitudes de frontières viennent compliquer singulièrement la question de la juridiction épiscopale ${ }^{4}$. Malgré ces amputations, le territoire administré par l'évêque de Québec demeure immense et, comme il se peuple de plus en plus en certains endroits, il faut songer à le diviser. Les jeux de la politique et de la diplomatie obligent à nommer d'abord des évêques auxiliaires et vicaires généraux en charge de certains districts (Montréal, Maritimes, Territoires du Nord-Ouest, Haut-Canada) avant de procéder à l'érection de véritables diocèses (1826: Kingston; 1829: Charlottetown; 1836: Montréal; 1841: Toronto; 1842: Fredericton). Mais c'est, en 1844, la formation d'une province ecclésiastique avec siège métropolitain à Québec et diocèses suffragants à Montréal, Kingston et Toronto - et bientôt à Terre-Neuve - qui pose vraiment le problème de la collégialité épiscopale ${ }^{5}$. On le résout alors par le mode traditionnel des conciles et des assemblées d'évêques.

\section{Les conciles provinciaux}

Tout au long des interminables tractations qui conduisent finalement à la création de cette province ecclésiastique revient régulièrement à la surface le problème de la collaboration entre les évêques pour uniformiser la discipline ecclésiastique et promouvoir les intérêts catholiques.

\footnotetext{
${ }^{4}$ Guy Arbour, Le droit canonique particulier au Canada, Ottawa, Ed. de l'Université d'Ottawa, 1957, pp. 37-40.

${ }_{5}^{5}$ Lucien Lemieux, L'établissement de la première province ecclésiastique au Canada, 1783-1844, Montreal, Fides, 1968, passim.
} 
Pour sa part, $\mathrm{M}^{\mathrm{gr}}$ Joseph Signay, évêque de Québec, ne voít qu'inconvénients et embarras dans les rencontres suggérées: difficulté de réunir en même temps des prélats si éloignés les uns des autres; longs retards dans l'expédition des affaires qui pourraient requérir l'avis de chacun; différence de moeurs et d'usages chez les fidèles des diverses régions, ce qui pourrait donner naissance à des incompréhensions entre les évêques ${ }^{6}$; il craint la réunion d'un concile aussi parce qu'il a peur que les prélats moins favorisés en profitent surtout pour lui quémander des prêtres et de l'argent, mais il voit un plus grand risque encore dans la possibilité que les évêques de langue anglaise deviennent majoritaires et qu'ils en arrivent à bouleverser l'antique discipline du diocèse de Québec ?. Fervents protagonistes de la création de la province ecclésiastique de Québec, les évêques de Montréal $-\mathbf{M}^{\mathrm{gr}}$ JeanJacques Lartigue et $\mathbf{M}^{\mathrm{gr}}$ Ignace Bourget $-\mathrm{y}$ voient plutôt l'occasion de rattacher à l'archevêché de Québec tous les diocèses de l'Amérique du Nord britannique, ce qui permettrait de reprendre un certain contrôle sur les territoires détachés de l'Église mère et d'y maintenir ou rétablir la discipline du diocèse de Québec menacée par la nomination d'évêques écossais ou irlandais qui ont tendance à s'inspirer des coutumes de leur pays; le métropolitain pourrait convoquer un concile et des réunions ordinaires qui ne pourraient qu'avoir des résultats bénéfiques:

Il y aura, prétend $\mathrm{M}^{\mathrm{gr}}$ Bourget, unité de conduite chez tous les évêques de la province, attachement plus grand aux saintes règles de l'Église, uniformité de discipline, plus de lumières dans leurs conciles, puisque l'Esprit-Saint s'est engagé à y présider plus de force dans le gouvernement plus de respect dans le clergé pour l'autorité épiscopale qui ne pourra plus être accusée de despotisme, plus de moyens pour l'élection de bons évêques [d'autres textes précisent qu'il faudra des évêques canadiens au lieu d'ecclésiastiques venant d'Irlande ou d'Écosse ], ce qui est essentiellement vital pour l'Église, plus de majesté et de pompe dans le culte, ce qui n'est pas peu de chose pour attacher les peuples à la religion et leur inspirer pour elle un profond respect, plus d'union entre les évêques dans leur rapport avec le gouvernement qui y regarderait plus d'une fois avant de se mettre à dos le corps épiscopal, si jamais il voulait gêner la religion ou adopter quelques mesures qui blesseraient des droits et privilèges, etc. ${ }^{8}$

\footnotetext{
${ }^{6}$ Ibid., p. 459.

${ }^{7} \mathrm{M}^{\mathrm{gr}}$ Signay à $\mathrm{M}^{\mathrm{gr}}$ Ignace Bourget, 1 janv. 1845, Archives de l'archevêché de Québec (AAQ), Registre des lettres, 21, p. 144.

${ }^{8} \mathrm{M}^{\mathrm{gr}}$ Bourget à $\mathrm{M}^{\mathrm{gr}}$ Signay, 24 av. 1841, cité dans Lemieux, op. cit. , p. 459.
} 
Quelques autres évêques de colonies britanniques sont du même avis que leurs confrères de Montréal, mais on notera que le travail collégial est surtout perçu comme un moyen d'aider les chefs religieux dans leurs fonctions.

Les réticences des évêques de Québec - tout en favorisant la création d'une province ecclésiastique, $\mathbf{M}^{\mathrm{gr}}$ Pierre-Flavien Turgeon a les mêmes objections que $\mathbf{M}^{\mathrm{gr}}$ Signay à propos d'un concile provincial retardent la tenue du premier concile provincial de Québec. Il a finalement lieu du 15 au 28 août 1851 et il sera suivi de six autres en $1854,1863,1868,1873,1878$ et 1886.

À plusieurs points de vue, le premier concile provincial de Québec, qui prend modèle sur ceux de Baltimore, trace la voie pour tous les autres qui suivent; pour le sujet qui nous intéresse, il est surtout un exemple de travail collégial. Non seulement chacun des évêques convoqués est-il invité à faire des suggestions à propos des questions à débattre, mais on l'invite à rédiger les textes qu'il aimerait faire voter et à tenir des réunions consultatives avec les prêtres de son diocèse. Bien plus, le concile lui-même est précédé d'une espèce de pré-concile, une réunion préparatoire tenue à Montréal en mai 1850, à laquelle sont invités tous les évêques de la province ecclésiastique de Québec - y compris celui de Terre-Neuve, mais non ceux des Maritimes qui sont pourtant invités au concile - et qui, en plus de préparer presque entièrement l'ordre du jour de la grande réunion projetée en copie le faste extérieur, le règlement et la procédure de travail ${ }^{9}$. Au concile luimême, tout se fera en équipe: évêques et théologiens se regrouperont en commissions, appelées aussi congrégations particulières; chaque commission présentera ses rapports aux congrégations générales, composées de tous les évêques et de tous les théologiens, et ces derniers seront appelés à donner leur avis; les évêques décideront ensuite en réunion privée ce qu'il faudra en retenir et formuleront ou modifieront les décrets qui, eux, seront proclamés dans des sessions solennelles et publiques. Puis le tout sera envoyé à Rome pour approbation ${ }^{10}$.

L'histoire des conciles provinciaux de Québec a été retracée avec minutie par Jacques Grisé et je n'ai pas l'intention de vous la résumer, même pour en extraire les thèmes et les lignes de force. Voici cepen-

\footnotetext{
9 Jacques Grisé, Les conciles provinciaux de Québec et l'Église canadienne (18511886), Montréal, Fides, 1979, pp. 31-40.

${ }^{10}$ Ibid., p. 66.
} 
dant quelques remarques générales concernant notre propos. Le premier concile est presque déjà un concile national (ou plénier) puisque seuls les évêques de la côte du Pacifique ne sont pas invités: sont convoquées, en effet, les autorités religieuses de Québec, Montréal, Kingston, Toronto, Terre-Neuve, du Nord-Ouest et de Bytown, comme rattachées à la province ecclésiastique et celles de Charlottetown, du Nouveau-Brunswick, d'Halifax et d'Arichat, d'après la bulle du 12 juillet $1844^{11}$. Cependant, dès le deuxième concile en 1854, les évêques des Maritimes, regroupés dans la province ecclésiastique de Halifax depuis 1852 , sont exemptés, de même qu'à partir de 1873, ceux des provinces ecclésiastiques de Toronto et de Saint-Boniface érigées respectivement en 1870 et 1871. Il faut noter aussi que des conciles provinciaux ont été tenus, sur-

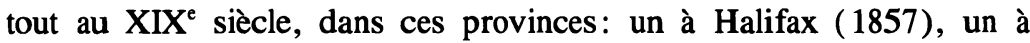
Toronto (1875), un à Saint-Boniface (1889) et un à Montréal (1895).

Malgré une présence des évêques de langue anglaise peu nombreuse et de courte durée, les conciles provinciaux de Québec révélent déjà un certain clivage entre francophones et anglophones et une première brèche dans l'idéal collégial. Le concile de 1868 en offre un exemple frappant. Une bonne partie des débats de l'assemblée générale des évêques tourne autour de l'important problème du nouveau découpage de la carte ecclésiastique canadienne. À cause de l'union fédérale de 1867 et de l'augmentation du nombre des catholiques, les autorités religieuses sentent le besoin d'établir un nouvel équilibre entre les diverses parties du nouveau pays. Le concile est ainsi saisi de plusieurs questions: doit-on diviser la province ecclésiastique de Québec qui s'étend encore jusque dans l'Ouest? Combien y aura-t-il désormais de provinces ecclésiastiques? quels diocèses composeront chacune des provinces? quelle sera la métropole en Ontario? Pendant trois jours, la congrégation générale des évêques consacre de longues séances à ces projets qui donnent lieu à des débats animés. Si les prélats sont unanimes à proposer la division de la province ecclésiastique de Québec, ce n'est qu'après de vives discussions qu'ils fixent le nombre de métropoles à trois, au lieu de quatre comme le demande l'évêque d'Ottawa. Quant à la composition de chacune des provinces, un seul problème se pose, mais d'envergure: le diocèse d'Ottawa demeurera-t-il rattaché à Québec? Il s'ensuit un affrontement "musclé" entre évêques anglophones et francophones; après des interventions passionnées de part et d'autre, seuls $\mathbf{M}^{\mathrm{gr}}$ John Farrell, de Hamilton, et $\mathbf{M}^{\mathrm{gr}}$ Edward John Horan, de Kingston, ne veulent pas consentir à laisser Ottawa dans la province ecclésiastique de Québec,

11 lbid., p. 59. 
mais la majorité doit faire preuve d'énergie pour empêcher, le lendemain, la remise en question du vote par les évêques de langue anglaise. Malgré la décision du concile, $\mathbf{M}^{\mathrm{gr}} \mathrm{John}$ Joseph Lynch, de Toronto, et ses suffragants font des réclamations à Rome et y font des mains et des pieds pour obtenir l'annexion de la partie ontarienne du diocèse d'Ottawa; seule la création de l'archidiocèse d'Ottawa en 1886 les empêche de réaliser leur rêve de rendre l'Ontario catholique homogène anglais ${ }^{12}$.

Une fois seuls entre eux, les évêques francophones ont-ils autant de difficultés à s'entendre? Oui, d'une certaine façon. Dans cette dernière partie du $\mathrm{XIX}^{\mathrm{e}}$ siècle, ils forment deux groupes qui pa:fois s'affrontent sur des sujets comme le rôle du clergé en politique, la question universitaire, le démembrement du diocèse de Trois-Rivières, la désignation de nouveaux évêques, etc. La division est moins idéologique - ils sont tous ultramontains de doctrine - que tactique et prend souvent racine dans des sensibilités différentes ou des liens profonds d'amitié. Les intransigeants vouent une admiration sans borne à $\mathrm{M}^{\mathrm{gr}}$ Bourget et veulent, immédiatement, pour l'Église du Québec, un rôle prépondérant dans presque tous les domaines; ils ont horreur des compromis. Les modérés, souvent accusés d'être catholiques libéraux, veulent tenir compte davantage de la réalité sociale et politique et, sous la direction de l'archevêque Elzéar-Alexandre Taschereau, prônent une politique de prudence et d'attente. Cependant, hormis des échanges vifs à l'occasion de l'étude de certains décrets - par exemple, en 1873, le décret sur le libéralisme catholique et celui sur la liberté de l'Église dans ses rapports avec le pouvoir civil - les conciles font voir davantage l'union des évêques que leurs divergences. Néanmoins, celles-ci influencent indirectement les réunions, puisqu'elles sont en partie responsables des retards apportés à la convocation des $6^{\mathbf{e}}$ et $7^{\mathfrak{e}}$ conciles (intervalles de 5 et de 8 années). Après celui de 1886, cessent les conciles provinciaux à Québec: la province civile compte maintenant deux sièges métropolitains, la discipline ecclésiastique est passablement unifiée dans tout le territoire, le cardinal Taschereau, vieilli et malade, cède l'administration à son coadjuteur, $\mathbf{M}^{\mathrm{gr}}$ Louis-Nazaire Bégin, qui ne se sent pas apte à faire la convocation... On sent moins le besoin de ces réunions grandioses et l'archevêque de Québec obtient facilement de Rome, de trois ans en trois ans, la dispense du concile.

\footnotetext{
${ }^{12}$ Pour plus de détails, voir: Grisé, op. cit., pp. 267-88; Nive Voisine, LouisFrançois Laflèche, deuxième évêque de Trois-Rivières, t. I, Saint-Hyacinthe, Edisem, 1980, pp. 122-25.
} 
Mais il y a plus. L'idée se répand de plus en plus de la nécessité d'un concile plénier pour l'ensemble du territoire canadien, un projet qui ne se réalise cependant qu'en 1909.

\section{Le concile plénier de Québec (1909)}

L'idée de réunir en concile tous les évêques du Canada remonte au moins à 1876 . $\mathbf{M}^{\mathrm{gr}}$ Lynch, de Toronto, en fait la proposition à $\mathbf{M}^{\mathrm{gr}}$ Taschereau dans une lettre du 21 avril de cette année et il renouvelle sa demande, le 10 mai 1877, cetie fois-ci auprès dú délégué apostolique, $\mathbf{M}^{\mathrm{gr}}$ George Conroy. Dans ce dernier texte, il livre le fond de sa pensée; après avoir critiqué les efforts des évêques du Québec pour tâcher d'influencer le monde politique et après avoir noté que ce qui est bon pour la partie anglaise du pays ne l'est pas toujours pour la partie française et vice versa, il ajoute: «après tout, le Canada français n'est qu'une province parmi quatre ou cinq»; il y a 12 prélats de langue anglaise,

ils ne sont que sept dans la Province de Québec. La Province de Manitoba est semblable à la nôtre, bataillant pour se tailler une place parmi les protestants. On ne doit pas sacrifier toutes les autres provinces aux intérêts du Québec, riche en ressources de toutes sortes, mais hélas en pleine confusion. ${ }^{13}$

Or, toute autre est l'opinion des évêques du Québec, tous regroupés pour une fois autour de l'archevêque Taschereau et de son successeur. D'une part, dans l'archidiocèse de Québec, soulignent-ils, les grandes questions disciplinaires ont été réglées et il n'y a plus «qu'à maintenir ce qui a été fait»; il resterait aux autres provinces ecclésiastiques à se mettre au diapason: «D'après ce que je connais, écrit $\mathbf{M}^{\mathrm{gr}}$ Taschereau, je crois que bien des affaires sont restées en arrière dans les autres provinces, mais alors ce serait à celles-ci à en faire les frais" [c'est-à-dire d'un recours à Rome et de l'envoi d'un nouveau délégué apostolique] ${ }^{14}$. D'autre part, l'Église québécoise se définit comme une Église nationale; elle se perçoit comme l'incarnation d'une nationalité conçue comme peuple élu par Dieu. La société est une nation et la nation est une Église, dont le clergé sera l'instance suprême définissant les projets et

${ }^{13} \mathrm{M}^{\mathrm{gr}}$ J.J. Lynch à $\mathrm{M}^{\mathrm{gr}} \mathrm{G}$. Conroy, 10 mai 1877, Archives de Propaganda Fide, Rome (APFR), Scritture riferite nei congressi (SRC), America Settentrionale-Canadà (ASC), 16 (1877A), f. 78r-81v.

${ }^{14} \mathrm{M}^{\mathrm{gr}}$ Taschereau à $\mathrm{M}^{\mathrm{gr}}$ Thomas Duhamel, 9 janv. 1879, AAQ, Registre des lettres, 32, pp. 504-6. 
les normes. Et dont il est le protecteur contre les ennemis qui l'entourent (les Juifs, les franc-maçons, les protestants, les Irlandais, «ces protestants déguisés»): «La race franco-canadienne, écrit $\mathbf{M}^{\mathrm{gr}}$ Taschereau, est entourée d'ennemis qui en different par la race, la langue et la religion; elle se maintient parcequ'elle [ sic] est unie sous son clergé" ${ }^{15}$. Il ne saurait donc être question pour elle de se perdre dans un grand tout canadien, si catholique soit-il. En conséquence, pendant très longtemps, l'Église catholique canadienne demeure un agrégat formé de deux blocs culturels: l'Église canadienne-française qui, à la fin du $\mathrm{XIX}^{\mathrm{e}}$ siècle, englobe les provinces ecclésiastiques de Québec, de Montréal, d'Ottawa et de Saint-Boniface, et l'Église canadienne-irlandaise, composée des provinces ecclésiastiques de Toronto, de Kingston et de Halifax. L'ethnie des évêques reflète bien cette dualité culturelle. Des 29 prélats (y compris les vicaires apostoliques et les coadjuteurs), 18 sont francophones et 11 anglophones. Les premiers sont d'origine canadienne-française, sauf les évêques de la province ecclésiastique de Saint-Boniface qui tous, à part $\mathbf{M}^{\mathrm{gr}}$ Adélard Langevin, viennent de France. Chez les anglophones, six évêques sont nés en Irlande et deux sont d'ascendance irlandaise. Sans doute, certains événements - la question des écoles du Manitoba, par exemple - rapprochent-ils suffisamment ces deux blocs pour qu'ils engagent parfois des actions communes, mais c'est rare et très laborieux.

Dans ce clivage culturel, nous avons une des raisons principales du retard apporté à la tenue du premier (et dernier) concile plénier canadien. Cependant, à mesure qu'approche le $\mathrm{XX}^{\mathrm{e}}$ siècle, de plus en plus d'évêques prennent conscience de sa nécessité pour corriger les divergences régionales et, comme le note $\mathrm{M}^{\mathrm{gr}}$ Bégin en 1899 , "pour réunir en un faisceau les forces dispersées de notre épiscopat" ${ }^{16}$. La Propagande elle-même se déclare favorable à l'idée et, par l'intermédiaire des délégués apostoliques Diodème Falconio et Donato Sbaretti, oblige les évêques canadiens à en commencer les préparatifs. De multiples obstacles, dont à partir de 1905 le peu d'enthousiasme de l'archevêque de Québec mais aussi la faiblesse des textes préliminaires qu'il faut recommencer, retardent d'année en année la convocation. Les interventions de $\mathrm{M}^{\mathrm{gr}}$ Sbaretti qui se montre trop empressé de présider les assises et qui, au dire des ultramontains intransigeants et des jésuites, est trop « entiché de tout ce qui est américain» et trop commis à l'égard de la suprématie de la langue anglaise, forcent la Propagande à recon-

${ }^{15}$ Le même à $\mathrm{M}^{\mathrm{gr}}$ Louis-François Laflèche, 23 avril 1879, ibid., 32, pp. 580-1.

${ }^{16} \mathrm{M}^{\mathrm{gr}}$ Bégin au card. Mieczyslaw Ledóchowski, 19 sept. 1899, ibid., 38, p. 27. 
naître qu'il appartient aux évêques de «déterminer le temps de la célébration du Concile et les matières qui doivent y être traitées" ${ }^{17}$. Telle n'est pas l'opinion du délégué et des évêques irlandais qui, en 1907, pressent toujours la tenue d'un concile, "sans que Rome nous en manifeste même le désir», constate $\mathbf{M}^{\text {gr }}$ Bégin. Cependant, l'opinion de $\mathbf{M}^{\mathrm{gr}}$ Sbaretti prévaut à Rome et, le 25 mars 1909 , le préfet de la Congrégation du Concile lui demande de convoquer tout l'épiscopat canadien à Québec en septembre 1909 et de présider ce concile plénier.

Le moins qu'on puisse dire, c'est que l'enthousiasme n'est pas délirant quand se réunissent à Québec les Pères conciliaires, mais ils font preuve d'une bonne volonté exemplaire. Ils sont quarante - huit archevêques, dix-neuf évêques, trois vicaires apostoliques, trois évêques auxiliaires, un préfet apostolique, cinq administrateurs de sièges vacants, un abbé mitré - à siéger du 10 septembre au ler novembre 1909, en compagnie de cent vingt-quatre théologiens, vicaires généraux et supérieurs de communauté. Ils s'affirment tous animés d'un même désir, "garder l'unité de la foi et l'intégrité des moeurs», et aussi d'un même espoir, «assurer l'union plus ferme des forces catholiques en notre pays". Ils s'imposent un rythme de travail infernal: cinq jours par semaine, les soirs et les fins de semaine étant réservés à des manifestations publiques (cortèges, conférences publiques, pèlerinages, réunions mondaines); ils réussissent ainsi à abattre une besogne considérable: ils proclament 688 décrets qui, après approbation par Rome, sont promulgués le 25 avril 1912 .

Le concile plénier de Québec a été considéré à l'époque comme un des grands événements de l'histoire de l'Église catholique canadienne. À distance, faut-il en nuancer la portée? L'ensemble des décrets constitue un traité de théologie pastorale et un code de conduite pratique. Le canoniste Valérien Bélanger y a vu «l'une des législations particulières les mieux faites dans l'Église». Les principes qui l'inspirent suivent de près l'ancien droit ecclésiastique et réflètent une orthodoxie inattaquable. L'entrée en vigueur, le 19 mai 1918, du Codex juris canonici, oeuvre de l'équipe du cardinal Gasparri, devait reléguer aux oubliettes ces décrets, sauf leur contenu dogmatique et une soixantaine de prescriptions particulières. On a dit avec justesse que le Codex a «rejeté dans l'ombre le concile avant qu'on ait eu le temps de le pratiquer". Par ailleurs, le concile a-t-il été un pas décisif vers le travail épiscopal collégial au

\footnotetext{
${ }^{17}$ Le même à $\mathrm{M}^{\mathrm{gr}} \mathrm{T}$. Duhamel, 7 mai 1906, AAQ, $321 \mathrm{CN}$, Diocèse d'Ottawa, II, 126.
} 
sein de l'Église canadienne? Certes, il a aidé l'épiscopat à sérier les problèmes, établir des priorités et mieux comprendre certaines questions d'une portée régionale, comme par exemple celle des Ruthènes catholiques. Il a aussi pris des mesures propres à faciliter le règlement de problèmes liés à l'immensité du pays, à la diversité culturelle et au pluralisme religieux; les décrets concernant l'étude de l'anglais et du français dans les séminaires $(166,179)$, le rapprochement oecuménique $(330,351,407-415)$, l'accueil des immigrants (332-334), l'uniformité du cathéchisme (313), l'incardination et l'excardination des prêtres (125) répondent à des besoins spécifiques. Mais le concile ne semble pas avoir rapproché les membres anglophones et francophones de l'épiscopat canadien. Au cours des rencontres informelles, on n'a pas réussi à dégager un consensus sur le problème à la racine de nombreux désaccords dans l'agir pastoral: l'avenir culturel du Canada. Et les évêques sont repartis dans leur régions poursuivre leur travail collectif au sein d'assemblées plus restreintes ${ }^{18}$.

\section{Les assemblées d'évêques}

En dehors des réunions solennelles, tels les conciles pléniers ou provinciaux, les évêques canadiens ont toujours senti le besoin de se rencontrer plus ou moins régulièrement pour étudier des problèmes communs ou prendre position en tant que groupe. Ils le font le plus souvent au niveau d'un archidiocèse, d'une province civile ou, plus rarement, de l'ensemble du pays. Pour les besoins de mon exposé, je ne retiens comme exemples que les réunions de l'épiscopat du Québec et de l'assemblée des archevêques du Canada.

\section{a) L'assemblée des évêques du Québec}

Très tôt au $\mathrm{XIX}^{\mathrm{e}}$ siècle, les évêques ont profité de diverses circonstances - les meilleurs exemples sont les funérailles ou les sacres d'évêques - pour se réunir d'une façon informelle et discuter de problèmes précis. Avant 1871, des prélats de diverses parties du pays participent à ces séances de travail: la même chose se produit à Rome en 1869-1870, lors du concile de Vatican I, mais les réunions de l'ensemble de l'épiscopat canadien sont peu nombreuses, puisque, très tôt, les évêques se regroupent selon la langue ${ }^{19}$.

\footnotetext{
${ }^{18}$ Lionel Lindsay, éd., Le premier concile plénier de Québec (10 septembre-1er novembre 1909). Québec, Impr. de "L'Événement", 1910, 306 p. Bruno Desrochers, Le premier concile plénier de Québec et le Code de droit canonique. Washington, The Catholic University of America Press, 1942, 186 p.

${ }^{19}$ N. Voisine, op. cit., I, pp. 134-7.
} 
Au Québec, c'est l'archevêque Elzéar-Alexandre Taschereau qui donne aux réunions de sa province ecclésiastique leur caractère permanent. Dès octobre 1871, en effet, pour tâcher de mettre fin aux remous soulevés par le Programme catholique et de réunifier l'épiscopat fortement divisé, il convoque tous les évêques et les invite à venir siéger en compagnie d'un théologien chacun. Les évêques de Québec, Montréal, Ottawa, Saint-Boniface, Saint-Hyacinthe, Trois-Rivières et Rimouski se rencontrent à Québec du 17 au 24 octobre 1871, dans une grande discrétion qui intrigue les journalistes habitués au faste des conciles provinciaux: "C'est une réunion strictement privée et on ne peut savoir ce qui s'y discute», note le Journal de Québec ${ }^{20}$. Le nouvel archevêque y fait preuve d'un leadership si évident et efficace que ses collègues tiennent à faire connaître «le bonheur qu'ils ont éprouvé à se rencontrer avec leur Archevêque» et ils souhaitent se réunir ainsi "au moins une fois l'an" ${ }^{21}$. Un secrétaire attitré - ordinairement, le dernier évêque sacré - rédige un procès-verbal consacré dans un registre spécial. À partir de 1875 , cette réunion revient deux fois l'an, car elle coïncide avec les rencontres du Comité catholique du Conseil de l'instruction publique où siègent tous les évêques du Québec; ils profitent de leur présence à Québec pour se réunir le soir ou, quand besoin est, une journée avant ou après les travaux du Comité catholique. Les évêques du Québec sont donc assurés de se rencontrer au moins deux fois l'an, mais ils le font ordinairement plus souvent, car l'archevêque profite encore des circonstances particulières pour convoquer des réunions spéciales.

Cette assemblée est un excellent instrument de concertation dans les intervalles de conciles provinciaux, et encore plus après 1886 quand ces derniers disparaissent et quand elle regroupe désormais les archevêques et évêques de toutes les provinces ecclésiastiques du Québec. On y aborde de nombreux problèmes théologiques, politiques, mais surtout disciplinaires, et on s'efforce de trouver des solutions communes qui, sans obliger juridiquement, lient chacun des participants. Au XIX siècle, on y parle beaucoup des relations entre l'Église et l'État; dans les années 1920, les évêques débattent les lois civiles qui touchent au droit de l'Église: législation concernant le mariage, le divorce, l'assistance publique, les écoles juives. Ils discutent de problèmes pastoraux: les missions, l'action sociale, le cinéma, de même que de l'application uniforme des directives pontificales et, bien sûr, de problèmes temporels tels la lutte antituberculeuse, la colonisation et le syndicalisme.

\footnotetext{
20 "L'assemblée des évêques", Le Journal de Québec, 20 oct. 1871, p. 2.

${ }^{21} \mathrm{M}^{\mathrm{gr}}$ Jean Langevin, [Procès-verbal de l'assemblée de la province ecclésiastique du Québec ], 25 oct. 1871, Archives de l'évêché de Trois-Rivières (AETR), Fonds Laflèche, A4, 2-1.
} 
Le style de travail dépend beaucoup de celui qui préside l'assemblée, l'archevêque de Québec. Obligé d'affronter un "saint têtu" comme $\mathbf{M}^{\mathrm{gr}}$ Bourget, $\mathbf{M}^{\mathrm{gr}}$ Taschereau s'aliène les sympathies de la partie intransigeante de l'épiscopat du Québec et son autoritarisme naturel, ajouté à un parti pris évident en faveur de l'université Laval, aboutit finalement, en 1876, à une coalition de tous ses suffragants qui lui passent pardessus la tête pour recourir à Rome où ils délèguent $\mathbf{M}^{\mathrm{gr}}$ Laflèche. L'intervention de la Propagande, particulièrement par l'envoi d'un délégué apostolique en 1877-1878, sauve le leadership de l'archevêque et lui permet de reprendre le contrôle de l'assemblée épiscopale; désormais, et même avant d'être nommé cardinal en 1886, il la dirige avec assurance et autorité, au point de s'attirer des remarques acerbes de certains collègues. En 1883, par exemple, l'évêque de Rimouski, $M^{\text {gr }}$ Jean Langevin, lui écrit:

Je proteste contre cette façon de procéder de haute main, et sans nous donner l'occasion de voir les pièces, ni de discuter les choses ensemble et de nous concerter en une action commune.

Cependant il est bien possible qu'après avoir pris communication des documents, j'en vienne à la même conclusion; mais je ne puis admettre que l'on veuille ainsi nous forcer à nous prononcer les yeux fermés et de confiance sans connaissance personnelle. ${ }^{22}$

C'est finalement l'usure occasionnée par l'âge et la maladie qui lui fait diminuer cette emprise sur l'assemblée des évêques et même à en céder la présidence à son coadjuteur, $\mathbf{M}^{\mathrm{gr}}$ Louis-Nazaire Bégin, au début des années 1890.

Malgré les réticences qui ont précédé sa nomination à Chicoutimi et, plus tard, son transfert à Québec, $\mathbf{M}^{\mathrm{gr}}$ Bégin prend très tôt un grand ascendant sur ses collègues et devient un chef incontesté et un porteparole prestigieux. Le rôle qu'il joue tout au long de la question des écoles du Manitoba, les dénonciations qu'il se permet de certains membres de la curie romaine et même de $\mathbf{M}^{\text {gr }}$ Rafael Merry Del Val, délégué apostolique, surtout les commentaires courageux qu'il fait de l'encyclique Affari Vos et qui auraient fait dire à $\mathbf{M}^{\mathrm{gr}}$ Merry del Val: «Je n'ai jamais vu traiter une encyclique du Pape dans aucun pays comme cette dernière a été traitée au Canada", tout cela, uni à une

\footnotetext{
${ }^{22} \mathrm{M}^{\mathrm{gr}} \mathrm{J}$. Langevin à $\mathrm{M}^{\mathrm{gr}}$ Taschereau, 30 juin 1883, Archives de l'archevêché de Rimouski (AAR), Registre spécial de correspondance, III, pp. 226-7.
} 
personnalité exceptionnelle, lui gagne la sympathie, l'estime et la confiance de tous ses confrères francophones et lui permet de refaire une quasi-unanimité autour de sa personne. L'assemblée des évêques acquiert sous sa direction une nouvelle personnalité. La collégialité y est davantage présente. Les discussions sont très libres et contribuent à façonner un esprit de corps et un style de pensée et d'action qui est particulier à l'épiscopat du Québec et qui se perpétue longtemps presque tel quel. Perçues à la Délégation apostolique et à Rome comme des «réunions régionales», ces rencontres sont, de fait, des réunions d'un corps épiscopal toujours conscient d'incarner le destin d'un peuple. Les évêques le savent et le proclament. Dans une lettre collective envoyée directement au pape, le 5 février 1929, ils ne craignent pas d'affirmer que «le peuple canadien-français existe et qu'il a le droit d'exister», de dénoncer les calomnies dont il est l'objet à Rome de la part des assimilateurs et de rappeler «qu'un peuple ne se supprime pas ainsi» ${ }^{23}$. Ils tiennent également à traiter directement avec Rome toutes les questions importantes et à y déléguer au besoin des représentants habilités à parler en leur nom. En 1909, par exemple, certains songent à installer $\mathbf{M}^{\mathrm{gr}}$ Louis-Adolphe Paquet dans la Ville éternelle, dans un couvent de religieux alliés, à le faire nommer consulteur de certaines congrégations, puis à en «faire le représentant des quatre provinces ecclésiastiques françaises"; on lui ferait donner le caractère épiscopal et on lui adjoindrait un secrétaire, "de sorte qu'il arriverait à prendre une grande influence et à tenir en échec les intrigues irlandaises». Le projet n'est pas mis à exécution, mais il indique bien les tensions qui existent entre les dirigeants des deux Églises catholiques canadiennes et la difficulté de vivre une collégialité pan-canadienne. Vingt ans après, en 1929, les mêmes tensions subsistent et les évêques du Québec rappellent à Pie XI: «Nous sommes également peinés de sentir une sorte d'antipathie envelopper notre peuple et ses chefs spirituels. Nous sommes particulièrement humiliés, à l'arrivée de chaque Délégué Apostolique et à la vacance de chaque siège épiscopal, de nous heurter aux mêmes méfiances et d'être obligés d'entreprendre les mêmes démonstrations".

\section{b) L'assemblée des archevêques du Canada}

Il se fait quand même certaines tentatives de rapprochement et de travail en commun. À partir de 1901, semble-t-il, les archevêques canadiens se réunirent une fois l'an, sous la présidence du délégué

\footnotetext{
${ }^{23}$ "Lettre collective des évêques du Québec au pape Pie XI», annexe au Procèsverbal de la réunion des évêques de la province civile de Québec, 6 fév. 1929.
} 
apostolique, pour passer en revue les problèmes de l'Église canadienne. $\mathbf{M}^{\mathrm{gr}}$ Duhamel, archevêque d'Ottawa, préférerait que tous les évêques participent à ces réunions; $\mathrm{M}^{\mathrm{gr}}$ Bégin y associe ses suffragants en leur demandant parfois des suggestions pour l'établissement de l'ordre du jour. Je ne saurais vous dire si ces rencontres ont vraiment eu lieu tous les ans ni comment elles se déroulaient, car je n'en ai pas encore trouvé les procès-verbaux. Elles devaient plutôt laisser à désirer sous plusieurs rapports, car, lors de la réunion des 6 et 7 novembre 1911, $\mathrm{M}^{\mathrm{gr}}$ Pellegrino-F. Stagni, récemment nommé délégué apostolique, propose un certain nombre de règles de procédure. Les réunions auraient lieu chaque année à Ottawa, le premier mercredi d'octobre. Les archevêques non disponibles à cette date pourraient se faire représenter. Un secrétaire permanent ferait connaître à l'avance l'ordre du jour préparé après consultation des archevêques qui, eux-mêmes, seraient tenus de consulter leurs suffragants. Il ferait parvenir, à tous les membres de l'épiscopat un procès-verbal de la réunion. L'abbé C. Lecoq, supérieur de Saint-Sulpice et ex-secrétaire du concile plénier, assumerait les fonctions de secrétaire. La réunion du 2 octobre 1912 semble avoir été tenue selon cette procédure; elle a été la seule.

L'interruption de ces réunions d'archevêques pose problème. $\mathbf{M}^{\mathrm{gr}}$ Bégin en attribue la disparition au fait qu'elles "nécessitent un voyage très long pour quelques prélats et fort dispendieux» ${ }^{24}$. L'explication semble incomplète. $\mathrm{Ne}$ peut-on pas supposer que la mésentente entre francophones et anglophones sur la question scolaire et la nomination des évêques, conjuguée à la diversité des problèmes d'une région à l'autre du pays, ont miné l'intérêt que les archevêques pouvaient avoir à se rencontrer? Quoi qu'il en soit, les problèmes communs subsistent et les ennemis extérieurs obligent à reprendre l'idée. Nommé cardinal le 26 avril 1914 et se considérant comme le primat de l'Église canadienne, même s'il n'en a pas officiellement le titre, $\mathbf{M}^{\mathrm{gr}}$ Bégin se préoccupe beaucoup de l'avenir du catholicisme au Canada. Durant la première guerre mondiale, les moeurs évoluent rapidement et l'institution du mariage semble remise en question en certains milieux. Plusieurs font pression pour que les législateurs assouplissent la loi sur le divorce. C'est là un problème du ressort du gouvernement fédéral et le cardinal Bégin ressent le besoin d'une action concertée de tout l'épiscopat canadien et l'utilité d'une structure administrative pour soutenir et orienter le travail commun. En 1921, il souhaite une réunion de tous

${ }^{24} \mathrm{M}^{\mathrm{gr}}$ Bégin au card. Gaetano de Lai, secrétaire de la Consistoriale, 21 mars 1916, AAQ, Registre des lettres, 49. 
les évêques du pays pour discuter «de questions religieuses bien importantes», notamment le divorce et le mariage. Il songe à une réunion annuelle de l'épiscopat canadien et à l'établissement d'un comité central permanent qui ferait un examen préliminaire des questions. Il s'ouvre de son projet au délégué apostolique, $\mathbf{M}^{\mathrm{gr}}$ Pietro Di Maria, et à l'archevêque de Toronto, $\mathrm{M}^{\mathrm{gr}} \mathrm{Neil} \mathrm{McNeil}$, arguant que «l'union compacte des évêques sera toujours une puissance formidable qu'aucun gouvernement ne saurait mépriser". Lors de leur réunion de l'automne de 1922 , les évêques du Québec étudient la question, mais ne réussissent pas à s'entendre sur "quelque chose de pratique». Le cardinal Bégin réduit alors son projet à une assemblée des seuls archevêques, telle qu'elle existait avant 1912, et il presse le délégué de la convoquer. Les choses traînent en longueur et le départ du délégué Di Maria, en 1926, les retarde davantage encore.

En 1927, l'archevêque de Toronto réclame à son tour une assemblée des archevêques mais les évêques du Québec lui font savoir que «dans les circonstances et avant l'arrivée du prochain Délégué, il n'y a ni lieu ni possibilité d'obtenir cette assemblée» ${ }^{25}$. Dès son arrivée cette année-là, $\mathrm{M}^{\mathrm{gr}}$ Andrea Cassulo prend sur lui de mener à terme ce projet: il forme un comité provisoire de quatre membres pour préparer une réunion plénière des évêques et archevêques canadiens. Le délégué suggère trois questions dont une sur la création d'un comité permanent de l'épiscopat canadien pour suivre les problèmes relatifs à l'immigration, à l'action sociale et aux sociétés catholiques laïques. La première réunion des archevêques et évêques du Canada et de Terre-Neuve a lieu à Québec, les 3 et 4 octobre 1928. Elle est présidée par le cardinal RaymondMarie Rouleau, successeur du cardinal Bégin. Au cours de cette rencontre, l'épiscopat refuse de créer un comité permanent des évêques sur le modèle de la National Catholic Welfare Conference aux États-Unis. Des évêques craignent «les dépenses additionnelles d'un organisme semblable, même réduit". D'autres savent que la Consistoriale s'oppose «à ce que l'on crée cette sorte d'organisme là où il n'en existe pas". On se rabat donc sur la formule d'un comité ad hoc qui fera rapport tous les cinq ans à une assemblée de l'épiscopat, quitte à ce qu'aient lieu des réunions spéciales si dix évêques en font la demande ${ }^{26}$. C'est peu, sans doute, mais c'est quand même la préfiguration de l'émer-

\footnotetext{
${ }^{25}$ Procès-verbal de la réunion des évêques de la Province civile de Québec, ler fév. 1927.

${ }^{26}$ Procès-verbal de la réunion des archevêques du Canada, 3-4 octobre 1928, AAR, Canada, 1928-1948.
} 
gence d'une structure de coordination. $\mathbf{M}^{\mathrm{gr}}$ Cassulo suggère, en 1932, de tenir ces réunions tous les trois ans. Il n'est pas entendu, mais les rencontres quinquennales de 1933 et de 1938 montrent qu'il pourrait être opportun d'en tenir plus fréquemment. L'action concertée commence à proter des fruits intéressants: par exemple, on avait pu régler la situation financière des diocèses de Regina, de Gravelbourg et de PrinceAlbert qui ne pouvaient rembourser les emprunts contractés auprès de la Sun Life, du Crédit foncier, de l'Alliance nationale et des banques. Si les longues tractations pour aboutir finalement à l'acceptation d'une réunion quinquennale montraient le peu d'intérêt de la majorité des évêques pour un travail collégial au niveau canadien, déjà les premiers résultats poussent à songer à une meilleure unification du leadership et de l'administration au sein de l'Église canadienne. C'est la guerre 19391945 qui contribuera le plus à faire aboutir ce projet.

\section{LE TRAVAIL COLLÉGIAL AU SEIN DE LA C.C.C.}

Le second conflit mondial, en effet, ne fait pas que favoriser les forces d'unification politique et culturelle, à l'oeuvre dans la Confédération canadienne, il fait prendre davantage conscience à la hiérarchie catholique de l'ampleur et de la complexité croissante des questions qui transcendent les frontières politiques des États provinciaux, les régionalismes et la juridiction des ordinaires, tels le communisme, les mariages mixtes, l'interventionnisme de l'État, l'Action catholique, les clubs neutres, la formation des prêtres, les missions, la représentation diplomatique auprès du Saint-Siège, etc. Bien plus, lors du plébiscite de 1942, les discussions qui entourent la préparation d'une lettre collective montrent encore davantage la nécessité d'une concertation. Le délégué apostolique, $\mathbf{M}^{\mathrm{gr}}$ Ildebrando Antoniutti, suggère de remplacer l'assemblée quinquennale des évêques par une réunion annuelle des archevê ques. Au printemps de 1942, l'épiscopat du Québec se rallie à l'idée d'une réunion annuelle des archevêques et d'une réunion quinquennale des évêques canadiens ${ }^{27}$. Les archevêques tiennent leur première réunion à Québec, les 28 et 29 octobre 1942. Le cardinal Jean-MarieRodrigue Villeneuve qui la préside soulève la question de la concertation de l'action: l'étendue du pays, la diversité des milieux et la différence des mentalités rendent problématique, dit-il, «une attitude uniforme de l'Épiscopat sur certaines questions d'intérêt général». Il

\footnotetext{
${ }^{27}$ Procès-verbal de la réunion des archevêques et évêques de la province civile de Québec, 5 mai 1942.
} 
suggère que les évêques canadiens siègent régulièrement en assemblée plénière et qu'entre les réunions un conseil de l'épiscopat veille à rendre uniformes leur pensée et leurs directives ${ }^{28}$. La proposition du cardinal est vague, mais elle pourra être améliorée grâce à deux modèles existants: la National Catholic Welfare Conference, née aux États-Unis après la première guerre, et les associations protestantes canadiennes qui «grâce à une organisation centrale pouvaient prendre une large part à la vie nationale du pays et obtenir en particulier des fonds pour défrayer leurs activités». Les archevêques élaborent donc un projet que les évêques discutent et précisent lors de leur réunion quinquennale tenue à Québec en octobre 1943. Ceux-ci s'entendent sur la mise à l'essai d'une structure qu'il suffira de roder au cours des ans. Elle comprendra: 1) une assemblée quinquennale de l'épiscopat canadien; 2) un conseil national où siègeront, une fois l'an, les archevêques et les évêques à la tête des commissions; 3) un comité exécutif composé du cardinal et de quatre archevêques; 4) neuf commissions permanêntes mandatées pour étudier, sous la présidence d'un évêque et avec le concours de théologiens et d'experts, des questions complexes à soumettre au conseil canadien; 5) un secrétariat. Commence alors la mise en place du nouvel organisme. Le comité exécutif tient sa première réunion à Québec le 15 décembre 1943. Il met sur pied le secrétariat permanent de l'épiscopat, dont le siège social sera à Ottawa et dont le fonctionnement sera supervisé par deux secrétaires, l'un francophone et l'autre anglophone. Le 26 septembre 1945, le conseil national amorce l'étude d'une constitution qui sera approuvée pour cinq ans par la Consistoriale en juin 1948 et par l'assemblée des évêques le 13 octobre de la même année. Selon ce texte, la Conférence catholique canadienne (C.C.C.) est une organisation volontaire des évêques canadiens vouée à l'avancement de l'Église et à la coordination de l'activité catholique au Canada. Ses rouages comprennent: 1) une assemblée annuelle des évêques; 2 ) un bureau d'administration composé ex officio des cardinaux et de l'archevêque de Québec, et de six évêques élus par l'assemblée; 3) six commissions chargées d'aviser les évêques et de donner suite aux décisions prises par l'assemblée et le bureau d'administration; 4) un secrétariat localisé à Ottawa et dirigé par deux secrétaires.

Instrument moderne beaucoup plus efficace que tout ce qui a existé auparavant, la C.C.C. s'est révélée, sans conteste, un organisme de vigilance, de consultation et de coordination qui a assuré une plus grande influence et un plus grand prestige à l'Église catholique cana-

\footnotetext{
${ }^{28}$ Procès-verbal de l'assemblée des archevêques du Canada, 28-29 oct. 1942.
} 
dienne. Tout en tenant compte d'une façon prudente de la dualité canadienne, avec double branche pour le secrétariat et les commissions et représentation anglophone et francophone égale au bureau d'administration, elle a permis l'élaboration de politiques d'ensemble, la promulgation de textes communs et, entre les Églises anglophones et francophones, une concertation fructueuse dans de multiples domaines allant de la liturgie à l'oécuménisme. Pour l'épiscopat du Québec - et, mutatis mutandis, pour celui des autres régions du pays - la C.C.C. a été un lieu où il a pu mieux veiller aux intérêts spécifiques des catholiques francophones répartis à travers le Canada. Elle a constitué aussi tout à la fois un horizon élargi et un mécanisme d'insertion dans les affaires canadiennes et internationales. Elle a aussi inspiré les méthodes de travail de l'épiscopat du Québec aux prises lui aussi avec des problèmes accrus et complexes (mais avec le danger de doubler les organismes de la C.C.C.).

Cependant, les évêques ont-ils appris, au sein de la C.C.C., à travailler de façon collégiale? Je n'ai malheureusement pas tous les éléments pour répondre d'une façon complète à cette question, mais une incursion dans la documentation issue de l'organisme, me permet d'en douter beaucoup. C'est, en grande partie, le mode de fonctionnement qui est en cause. Par bien des aspects, la C.C.C. a eu tendance à devenir une petite curie romaine au sein de laquelle les cadres permanents et les experts ont pesé lourds dans les orientations et les décisions que prenait l'épiscopat. Ce n'est guère surprenant quand on songe aux réticences qui ont précédé sa naissance, qu'elle a été une superstructure mal articulée aux chancelleries diocésaines et aux assemblées épiscopales régionales, que les hommes qui composaient son Assemblée plénière se connaissaient peu, qu'ils communiquaient difficilement à cause de la disparité linguistique, qu'ils n'avaient pas l'habitude du travail en équipe ni, ne l'oublions pas, les mêmes idées sur le Canada et l'insertion de l'Église dans un pays aussi diversifié.

Ce diagnostic a été posé, dès 1964 , donc pour les 20 premières années d'existence de la C.C.C., par l'abbé Charles-E. Mathieu, à titre de nouveau secrétaire général. Voici quelques extraits de cette analyse lucide et prophétique:

Je constate jusqu'ici que les Évêques font trop confiance à leurs "permanents" en ce sens qu'Ils s'en remettent presque sans étude ou discussion à leurs recommandations. [...] La conférence, dans sa structure actuelle, n'est pas assez démocratique; et c'est peutêtre pour cela que plusieurs Membres ne s'y engagent pas? [...] les Évêques - pas plus que les médecins, avocats, chefs d'entre- 
prises, etc. - n'ont eu la chance d'apprendre à travailler en équipe, à s'affronter, à discuter à l'aise, à parlementer... Ills devront l'apprendre à la C.C.C., coûte que coûte, à leurs risques et dépends... [... La collégialité aura beau être définie en termes de théologie ou de droit canon, elle devra en définitive s'exprimer dans les faits et la réalité psycho-sociale quotidienne. Le dialogue s'apprend et se pratique. Le travail d'équipe aussi... [... Enfin viendra l'heure de la véritable planification et action d'ensemble. [...] Au moment de l'action concertée apparaîtra alors, selon toute probabilité, l'opportunité de "décentraliser" la $\mathrm{CCC}$ et de réévaluer ses services en relation avec ceux des chancelleries diocésaines, de l'assemblée épiscopale du Québec et du Manitoba (les seules qui existent et fonctionnent actuellement). ${ }^{29}$

Ces propos ne tombent pas dans l'oreille de sourds et, dès 1965, commence un immense travail de mise à jour. En 1966-1967, le diligent abbé Mathieu fait parvenir aux évêques un très volumineux ( 7 documents, 748 pages) Rapport sur l'évaluation et la réorganisation de la C.C.C.. Dès lors s'enclenchent toute une série de réformes dont le point culminant sera, en 1973, la régionalisation de la C.C.C., qui deviendra en 1977 la C.E.C.C. (Conférence des évêques catholiques du Canada). C'est une tentative pour accroître une collégialité fragile en donnant de nouveaux pouvoirs aux unités pastorales de base, les secteurs apostoliques. La C.C.C. y est fortement poussée par l'A.E.Q. qui a elle aussi entrepris sa réforme et qui, dès 1971, désire devenir une région ecclésiastique, dans le sens indiqué par Christus Dominus, à cause «d'une solidarité vécue depuis cent ans" et de la nécessité de bâtir une pastorale homogène, ancrée dans les réalités socio-culturelles ${ }^{30}$. Tout n'est pas réglé définitivement, puisque, encore en octobre 1982, les évêques se permettent, publiquement, une séance de défoulement collectif rapportée par la presse. Une intervention, celle de $\mathrm{M}^{\mathrm{gr}}$ Alexander Carter, de Sault SainteMarie, est particulièrement remarquée:

«On a tourné autour du pot assez longtemps et c'est le temps de se parler ouvertement et franchement», a soudain déclaré au micro un évêque ontarien anglophone, originaire du Québec, $\mathbf{M}^{\mathrm{gr}}$ Alexander Carter, de Sault-Sainte-Marie. «Je trouve que les évêques du Québec ne sont pas une minorité ici, a-t-il poursuivi. S'ils se sentent un peu dépaysés au sein de la CECC, c'est peut-être qu'ils

\footnotetext{
${ }^{29}$ Charles-E. Mathieu, Les dix prochaines années de la CCC, 9 mai 1964, 2 p.

${ }^{30}$ Procès-verbal de la réunion des archevêques et évêques de la province civile de Québec, 15-16 sept. 1971.
} 
ne sont pas à leur aise lorsqu'ils sont en dehors du Québec. Après avoir entendu ce qu'il s'est dit sur les frustrations de certains évêques québécois dans notre organisme, je crois qu'il faut et que nous devons nous poser la question: est-ce que nous voulons une ou deux conférences épiscopales canadiennes? Quant à moi, je serais très humilié et très découragé si nous devions en venir à nous scinder en deux conférences. Quelle image de l'Église projetterions-nous, en effet, si les évêques du Québec se séparaient de la CECC?... Je crois que le problème auquel nous faisons face présentement n'est pas un malaise, c'est une maladie. Et cette maladie, ne sera pas guérie par des voeux pieux...» ${ }^{31}$

\section{CONCLUSION}

De ce premier survol de l'activité collégiale des évêques canadiens, je retiens les éléments suivants qui nuancent mon hypothèse de départ:

1) L'épiscopat canadien a un long passé de travail collégial. Que ce soit dans les conciles ou dans les assemblées d'évêques, les prélats ont partagé leurs idées et pris des décisions communes, surtout dans les domaines doctrinaux et disciplinaires. Il ne faut pas se surprendre, cependant, si ce travail collégial ne correspond pas totalement à la vision de collégialité développée par Vatican II.

2) Malgré certaines lacunes, la collégialité épiscopale se vit mieux dans les regroupements d'unités de base homogènes et d'un milieu socioculturel rapproché: les assemblées régionales et, au Québec, les inters. Cette collégialité demeure fragile: ce sont des hommes qui composent ces organismes, et des hommes de valeur, doués de riche personnalité habitués à diriger et à commander, pour qui, avouons-le, il n'est pas facile de sacrifier une partie de leur autonomie au profit d'une intégration à un ensemble. Déjà en 1966, l'abbé Mathieu le soulignait: "De tous les types d'association, [les groupements de dirigeants et de chefs] sont en effet habituellement les plus difficiles à organiser, à maintenir et à développer ${ }^{32}$.

\footnotetext{
${ }^{31}$ Le Soleil, 28 oct. 1982, p. D-20.

${ }^{32}$ C.-E. Mathieu, Évaluation et réorganisation de la Conférence catholique canadienne, t. I, p. 32.
} 
3) Enfin, malgré des efforts sincères et de nombreux aspects positifs de la CCC et de la CECC, ${ }^{33}$ au niveau canadien la collégialité a été acquise péniblement, elle m'apparait encore superficielle et fragile et elle demeurera telle, je crois, tant que l'Église sera insérée dans une société politique elle-même divisée et quasi ingouvernable.

\author{
Nive VoIsINE \\ Département d'histoire \\ Université Laval
}

${ }^{33}$ L'abbé Mathieu les résument ainsi : «la participation active et l'assiduité des Membres aux réunions, aux consultations, aux votes; la nature généralement démocratique des délibérations; la bienveillance mutuelle des relations et des communications entre les Membres; le biculturalisme et le bilinguisme exemplaires qui imprègnent toute la vie de la Conférence; l'organisation méthodique; l'aide financière considérable aux diocèses et aux oeuvres; le nombre élevé de personnes et d'organismes déjà à l'oeuvre au service de l'Épiscopat, et cela bien avant que le Concile ne vienne consacrer officiellement l'existence et le rôle des conférences épiscopales; etc. etc. " (Ibid., p. 33). 